\title{
LA DINAMICA DE LA MANUFACTURA, EL CASO MEXICANO EN EL PERIODO 1995:01-2012:06
}

MANUFACTURING DYNAMICS, THE MEXICAN CASE, 1995:01-2012:06

\section{JORGE LUDLOW WIECHERS*}

Universidad Autónoma Metroplitana Azc.

\begin{abstract}
Manufacturing exhibits nonlinear trends in production levels, one being an active state and the other a passive state. We use a Markov model to estimate these two states, which are tied to United States imports of Mexican products. A regression is made in order to estimate whether there is an expansion or contraction in order to show which sectors have a greater propensity to expand or to decrease, there are several cases where the contracting level of output lasts longer than the expansionary alternative. This method of analysis can be applied to any Latin American economy.
\end{abstract}

Keywords: Manufacturing, Markov switching model.

JEL Classification: L16, L25, L52, L60.

\section{Resumen}

La manufactura tiene alteraciones no lineales en los volúmenes producidos, lo cual crea un nivel activo y otro pasivo. Se utiliza un modelo de Markov para estimar estos dos estados, los cuales están ligados a las importaciones de los Estados Unidos de productos mexicanos. Se hace una estimación de su crecimiento/contracción, mostrando cuáles son los sectores con una propensión a expandirse y cuáles con persistencia a decrecer, existiendo varios casos donde la baja productiva dura más tiempo que sus momen-

* Departamento de Economía, Universidad Autónoma Metropolitana Azc., México. E-mail: jlw@ nechikali. azc.uam.mx 


\begin{abstract}
tos de crecimiento. Este método de análisis se puede aplicar a cualquier economía latinoamericana.
\end{abstract}

Palabras clave: Manufactura, regímenes de Markov.

Clasificación JEL: L16, L25, L52, L60.

\title{
1. INTRODUCCION
}

Una actividad fundamental en el proceso de desarrollo de cualquier economía es la producción manufacturera, indicador ideal para revisar la actividad productiva mes a mes, ya que para el PIB solo hay informes trimestrales y anuales. El sector manufacturero contiene subsectores de suma importancia: alimentos, textiles, productos derivados del petróleo y del carbón, química y plásticos, transporte, aparatos eléctricos, etc. Cada uno de estos subsectores presenta un dinamismo propio, pudiendo suceder que un sector esté en expansión mientras que otro esté contrayéndose. Este trabajo presenta un método general, usando regímenes de Markov, para estimar los tiempos de crecimiento/contracción y las probabilidades de cambio de fase (crecer a decrecer, o al revés). Con este método se pueden responder estas preguntas claves: bajo el régimen de crecimiento o contracción ¿cuáles son sus tasas medias de cambio?, ¿en cada uno de los componentes de las manufacturas las caídas duran menos tiempo que las expansiones?

De los estudios sobre la evolución de la manufactura sobresale el de Unger (1993), quien hace notar que el desempeño exportador mexicano se caracteriza por una alta concentración, donde las exportaciones importantes se concentran en un número reducido de productos, en las que sus exportaciones las realizan un puñado de empresas relativamente grandes; el destino de la mayoría de las exportaciones se concentra muy notoriamente en un solo país: Estados Unidos. Entre sus recomendaciones de política económica sugiere que es prioritario tomar la senda del crecimiento exportador, de modo que a cada industria corresponda una política distinta según la fase de su evolución. Sin embargo, Fuji (2000), analizando manufacturas por sus componentes de maquila y no-maquila, encuentra que la causa básica de la dinámica de la manufactura es el desempeño del sector externo, el cual obliga a realizar ajustes en el nivel y tiempos de producción manufacturera. Fuji opina que es necesario generar una estructura industrial interna que esté integrada; sin embargo, no hace el análisis detallado por subsectores diferenciándolos, como está en el trabajo de Unger.

Posteriormente, Moreno y Palerm (2001) estudian, en términos agregados, el nivel del volumen del comercio y observan que el aumento del índice de comercio intraindustrial (usando el Indice de Grubel-Lloyd) es el resultado de políticas de gobierno. En el mismo sentido está el trabajo de Acevedo (2002), quien afirma que una contracción en el sector maquila de exportación se debe a una contracción de la demanda externa. Posteriormente, observando la crisis mundial, Dussel (2009) pone en duda incluso la viabilidad del modelo manufacturero. 
Otros estudios indican que la explicación del comportamiento del ciclo económico se debe a la relación entre México y los Estados Unidos. Mendoza (2008) y Mejía-Reyes y Campos-Chávez (2011) afirman que a partir del Tratado de Libre Comercio (NAFTA, North American Free Trade Agreement, 1994) los ciclos económicos se han sincronizado. Ambos trabajos analizan las regiones geográficas de México y confirman que los ciclos están sincronizados, ya que la producción es procíclica con la demanda exterior. Esta sincronización se explica por la integración vertical de los procesos productivos, resultante de la internacionalización de la producción de las empresas transnacionales. Los resultados son concordantes con la observación inicial de Unger (1993), en el que la manufactura manifiesta una alta concentración en cuanto a líneas de productos y su destino, pero también respecto de localidades geográficas.

Cuadra (2008) analiza las principales regularidades empíricas del ciclo desde 1980 hasta 2006, divide su estudio en un período de alta volatilidad, 1980-1998, y otro de estabilidad económica, 1999-2006. Él concluye que "el grado de sincronización entre el ciclo en México y el ciclo en los Estados Unidos es mucho mayor en el período más reciente", conclusión que refuerzan estudios anteriores.

Existen alternativas para analizar la dinámica de la manufactura. Una es vía el análisis insumo-producto, que busca sectores claves y clústeres productivos. Otra es vía crecimiento económico: productividad de los factores productivos, eficiencia técnica, retornos a escala, contabilidad del crecimiento, etcétera.

Así, en un mundo global, hablar de sincronización productiva ya no es suficiente, hay que tomar en cuenta cada uno de los componentes de la manufacturas y reconocer que los períodos expansivos y de contracción de estos componentes pueden ser diferentes. Al respecto hay solo unos pocos estudios en etapas muy iniciales que tocan este tema. Se requiere de una metodología general para entender la complejidad de la evolución de la manufactura en Latinoamérica, por lo que el objetivo de este artículo es desarrollar esta metodología para el caso mexicano. Veremos que este estudio considera una aproximación novedosa que nos permite mirar los problemas del desarrollo desde una nueva perspectiva.

Como se discutirá en el artículo es compleja la dinámica de la producción. El volumen de la producción afecta directamente el nivel de inventarios, el empleo, el capital de trabajo y el uso de la planta productiva. Así, caídas en la producción generan dudas sobre la necesidad de ampliar la capacidad instalada. Por ejemplo, en una contracción se vuelve difícil promover innovaciones en la línea de producción, incrementar el número de trabajadores y buscar capacitación para utilizar la maquinaria robotizada importada. De esta manera, este trabajo hace un análisis de las fases del ciclo económico de la manufactura en términos agregados y de todos sus subsectores (21 subsectores), para responder las siguientes preguntas:

Si un subsector crece, ¿qué probabilidad tiene de que continúe creciendo?, ¿por cuánto tiempo?

¿Cuál es la probabilidad de que un subsector estando en una expansión inicie la contracción?

Si el subsector se contrae, ¿qué tan probable es que continúe el descenso?, ¿por cuánto tiempo?

¿Cuál es la probabilidad de que se inicie el crecimiento cuando se enfrenta una caída? 
Cuando crece un subsector, ¿cuál será la tasa media de expansión?

Cuando decrece un subsector, ¿cuál será la tasa media de contracción?

En el largo plazo, ¿la probabilidad de crecer es alta y la probabilidad de decrecer es más baja?

El trabajo destaca el hecho de que una disminución en las compras de los Estados Unidos induce una contracción cuando se presenta una caída en las ventas, hecho que es consistente con la literatura general. Al respecto, en este estudio se utilizan datos publicados por la Reserva Federal (http://research.stlouisfed.org/fred2/) y del Instituto Nacional de Estadística, Geografía e Informática (INEGI http://www.inegi. org.mx/sistemas/bie/) para el período 1995:01-2012:06.

Este trabajo utiliza un modelo no lineal de regímenes de Markov. En el desarrollo de la metodología queda claro que se puede aplicar para la manufactura de cualquier país. En la Sección 2 se presentan y comentan las estadísticas básicas, junto con un análisis de cointegración entre la producción industrial de México y el comercio con los Estados Unidos. En la Sección 3 se explica el modelo. Los detalles más técnicos se presentan en el Anexo B. Luego, en la sección 4 se presentan los resultados. Al respecto, en el Anexo A se presentan los datos usados en este estudio. En la última Sección se presentan las conclusiones, así como las recomendaciones de política económica.

\section{ANALISIS PRELIMINAR DE LOS DATOS}

Una pregunta usual en esta área es si la relación de un mercado local con un mercado más grande es valiosa para el mercado más pequeño. Una respuesta inicial se puede obtener analizando las tasas de crecimiento de la manufactura y las tasas de cada uno de sus 21 subsectores con el comercio de los Estados Unidos.

La institución mexicana que publica las cifras económicas es el Instituto Nacional de Estadística, Geografía e Informática (INEGI). Para elaborar el índice de volumen físico de la producción manufacturera, el INEGI tiene una metodología compuesta por 21 subsectores. Para realizar el análisis tomamos la tasa de crecimiento mes a mes $\% Y_{t}=100 *\left(\log \left(Y_{t}\right)-\log \left(Y_{t-1}\right)\right)$, de cada una de las 22 series que publica INEGI (Tabla 1).

De la Tabla 1, podemos inferir la gran heterogeneidad entre sectores:

A) Los subsectores presentan diferentes tasas, tanto positivas como negativas. Por ejemplo, en el subsector Textil solo los Insumos textiles tienen una tasa anual positiva, pero muy baja. Por otra parte, Muebles tiene una cifra negativa. En cambio, la tasa más elevada corresponde a Transporte, que incluye automóviles, camionetas, camiones y tractocamiones, así como sus refacciones, componente tradicionalmente importante por sus exportaciones a los Estados Unidos.

B) En la desviación estándar se observa algo similar; el sector Textil junto con Cuero, Madera, Impresión, Maquinaria, Electrónicos, Transporte y Muebles presentan una variabilidad mayor que los demás subsectores, lo que indica alta volatilidad en la producción. 
TABLA 1

TASAS DE CRECIMIENTO DE LA MANUFACTURA Y SUS SUBSECTORES

\begin{tabular}{|lrrrrr|}
\hline Variable & Media & \multicolumn{1}{c}{ Anual } & Desv. Est. & Asimetría & Curtosis \\
\hline Manufactura & 0,243 & 2,960 & 4,757 & $-0,002$ & $-0,087$ \\
Alimentos & 0,138 & 1,665 & 3,595 & 0,059 & 0,012 \\
Beb. y Tab. & 0,336 & 4,104 & 7,843 & 0,613 & 0,653 \\
Ins. Textil & 0,077 & 0,930 & 10,230 & $-0,900$ & 0,732 \\
Prod. Textil & $-0,063$ & $-0,752$ & 13,929 & $-0,229$ & 2,329 \\
PrendasVestir & $-0,006$ & $-0,071$ & 10,678 & $-0,907$ & 3,041 \\
Cuero & 0,059 & 0,705 & 11,206 & $-0,758$ & 0,521 \\
Madera & 0,136 & 1,645 & 13,275 & $-0,300$ & $-0,386$ \\
Papel & 0,336 & 4,113 & 5,898 & 0,365 & $-0,859$ \\
Impresión & 0,267 & 3,255 & 12,389 & $-0,276$ & 0,063 \\
Petróleo & 0,070 & 0,844 & 6,500 & $-0,134$ & 2,262 \\
Química & 0,153 & 1,848 & 6,718 & $-0,004$ & $-0,164$ \\
Plástico & 0,350 & 4,276 & 8,305 & 0,330 & 0,246 \\
No Metálicos & 0,172 & 2,088 & 4,996 & 0,063 & 0,491 \\
Metal. Básica & 0,239 & 2,904 & 6,024 & 0,088 & 2,692 \\
Prod. Metálicos & 0,304 & 3,706 & 6,494 & $-0,406$ & 1,211 \\
Maquinaria & 0,229 & 2,788 & 10,506 & $-0,555$ & 1,593 \\
Electrónicos & 0,191 & 2,313 & 10,305 & $-0,285$ & 0,179 \\
Equip. Transporte & 0,281 & 3,430 & 9,016 & $-0,087$ & $-0,224$ \\
Transporte & 0,536 & 6,627 & 11,408 & $-0,300$ & $-0,191$ \\
Muebles & $-0,086$ & $-1,022$ & 15,916 & $-0,957$ & 5,468 \\
Otras Manuf. & 0,311 & 3,799 & 8,186 & 0,096 & 2,646 \\
\hline
\end{tabular}

Fuente: INEGI.

C) Uno esperaría ver mayoritariamente subsectores con tasas de crecimiento positivo que negativo, por lo que la asimetría debiera ser positiva. Sin embrago, esta asimetría es negativa en varios subsectores: Manufactura, Textiles, Cuero, Madera, Impresión, Petróleo, Química, Productos Metálicos, Maquinaria, Electrónicos, sector Transporte y Muebles.

D) El exceso de curtosis mide si las colas están más anchas que la distribución normal, esto es, si la variable toma valores extremos con más frecuencia que la distribución normal. En la Tabla 1 se observa que hay subsectores con asimetría negativa y un exceso de curtosis elevado. Esto sugiere que hay subsectores donde se presentan elevadas tasas de crecimiento negativo, como es el caso de Prendas de Vestir y Muebles.

En suma, las estadísticas básicas sugieren subsectores de producción industrial con bajo crecimiento y alta volatilidad y sectores con problemas importantes (crecimiento negativo).

Otro tema a considerar en la dinámica de la producción industrial mexicana es la relación entre esta producción y las caídas de las importaciones de los Estados Unidos provenientes de México (mostrada por bandas verticales grises en la Figura 1). Si las 
ventas a los Estados Unidos disminuyen, se observa una caída en las manufacturas mexicanas. Además, calculando la tendencia de la producción industrial por medio del filtro de Hodrick-Prescott (HP), se observa que las compras de los Estados Unidos inciden en la evolución o tendencia de la producción industrial.

\section{FIGURA 1}

LINEAS VERTICALES CUANDO DISMINUYEN LAS VENTAS A LOS EE.UU.

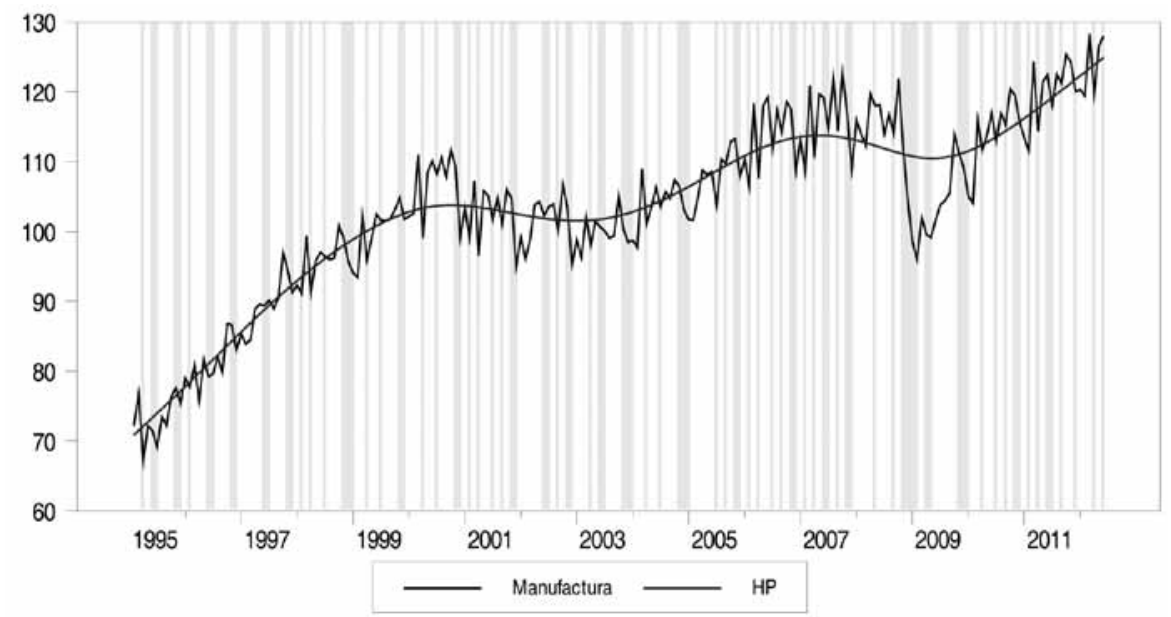

Fuente: Cálculos del autor.

Otra aproximación sencilla para ilustrar el punto anterior es mirar el índice de la producción industrial con las importaciones de los Estados Unidos de productos mexicanos (en tasas de crecimiento, Figura 2). Se toma como fecha de inicio 1995:01, ya que el año anterior se inició el Tratado de Libre Comercio entre México y los Estados Unidos.

La recta de regresión simple de la Figura 2 está dada por: Manufactura $\%=$ $0,506 \cdot$ Ventas $\%$, lo cual significa que $1 \%$ de incremento en las ventas a Estados Unidos se traduce en $0,506 \%$ de incremento en la actividad manufacturera.

Un análisis de cointegración permite llegar a una conclusión más definitiva sobre la relación entre la producción industrial de México y las importaciones de los Estados Unidos. Un incremento en la producción industrial de los Estados Unidos conlleva un aumento en los insumos requeridos desde México, con lo que se recibe un flujo de divisas por las transacciones realizadas. Nuestros resultados indican que estas tres variables están cointegradas: la Producción Industrial de los Estados Unidos (PRODINDEU), las Ventas a Estados Unidos (IMPMX) y la Manufactura (Manuf). 


\section{FIGURA 2}

\section{TASAS DE CRECIMIENTO DE LA PRODUCCION INDUSTRIAL EN MEXICO Y LAS IMPORTACIONES DE LOS ESTADOS UNIDOS (MX) A MEXICO}

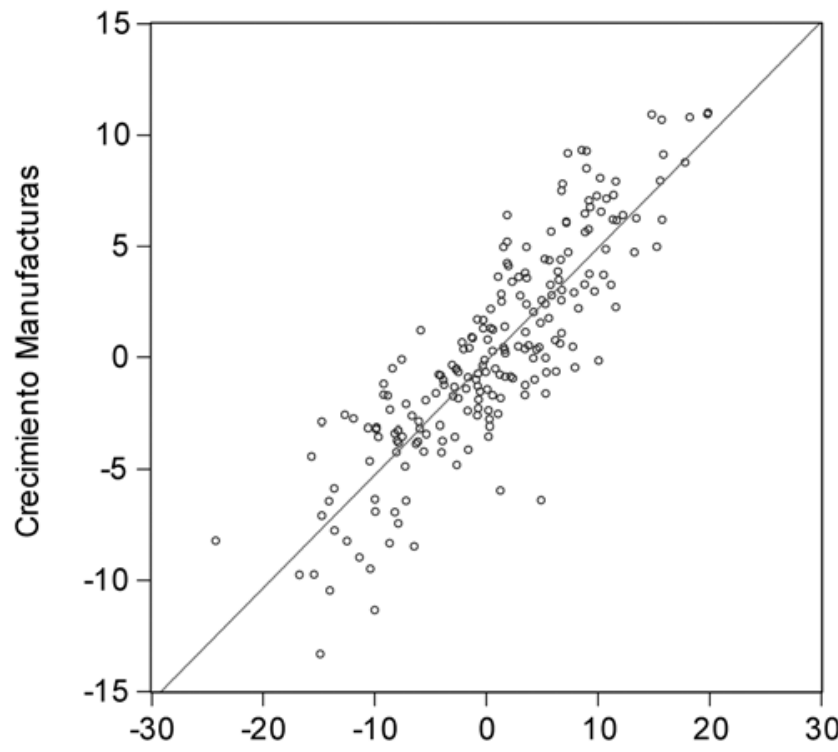

Crecimiento Ventas de Mx a los EE.UU.

Fuente: Cálculos del autor.

Para conseguir esta conclusión, se hace la prueba de Dickey-Fuller aumentado al nivel de significancia del 5\%, con 4 rezagos y con un valor crítico de $-3,431368$. Los resultados son los siguientes:

H0: Producción Industrial EU tiene una raíz unitaria

t-Statistic

Augmented Dickey-Fuller

H0: Ventas a EU tiene una raíz unitaria Augmented Dickey-Fuller

H0: Manufactura tiene una raíz unitaria Augmented Dickey-Fuller
$-2,546282$

t-Statistic

$-2,239347$

t-Statistic $-3,113496$
Probabilidad 0,3057

Probabilidad 0,4649

Probabilidad 0,1059 
Cada una de las tres series tiene una raíz unitaria. Como las variables son I(1): podemos proceder a buscar cointegración entre ellas, para la muestra que va desde 1995:01 a 2012:06. Los estadísticos de la traza y el valor propio máximo se indican a continuación en la Tabla 2. Ambos estadísticos señalan que hay una ecuación de cointegración, con un rezago.

\section{TABLA 2}

PRUEBA DE COINTEGRACION

\begin{tabular}{|c|c|c|c|c|}
\hline \multicolumn{5}{|c|}{ Prueba de la Traza } \\
\hline $\begin{array}{l}\text { Hipótesis nula } \\
\text { Número de } C E(s)\end{array}$ & Eigenvalue & $\begin{array}{l}\text { Estadístico } \\
\text { de la traza }\end{array}$ & $\begin{array}{c}0,05 \\
\text { valor crítico }\end{array}$ & prob.** \\
\hline Ninguna $*$ & 0.166936 & 54.90802 & 42.91525 & 0.0021 \\
\hline 1 a lo sumo & 0.056884 & 16.55263 & 25.87211 & 0.4490 \\
\hline 2 a lo sumo & 0.020052 & 4.253697 & 12.51798 & 0.7052 \\
\hline \multicolumn{5}{|c|}{ Prueba de valor propio máximo } \\
\hline $\begin{array}{l}\text { Hipótesis nula } \\
\text { Número de } \mathrm{CE}(\mathrm{s})\end{array}$ & Eigenvalue & $\begin{array}{c}\text { Estadístico } \\
\text { Valor propio } \\
\text { máxc }\end{array}$ & $\begin{array}{c}0,05 \\
\text { valor crítico }\end{array}$ & prob.** \\
\hline Ninguna* & 0.166936 & 38.35540 & 25.82321 & 0.0007 \\
\hline 1 a lo sumo & 0.056884 & 12.29893 & 19.38704 & 0.3878 \\
\hline 2 a lo sumo & 0.020052 & 4.253697 & 12.51798 & 0.7052 \\
\hline
\end{tabular}

Fuente: Cálculos del autor.

Nota: $\mathrm{CE}=$ relaciones de cointegración.

La ecuación de cointegración tiene la siguiente forma (ecuación (1)):

$\mathrm{EC}=\mathrm{PRODINDEU}(-1)+0,0014 * \operatorname{IMPMX}(-1)-1,1598 * \operatorname{MANUF}(-1)+1,5547$

Considerando el criterio de información de Akaike, el modelo adecuado en primeras diferencias es (ecuaciones 2-4):

$\mathrm{D}($ PRODINDEU $)=-0,0307 *($ PRODINDEU $(-1)+0,0014 * \operatorname{IMPMX}(-1)-1,1598 *$ MANUF $(-1)+1,5547)+0,2405 * \mathrm{D}(\operatorname{PRODINDEU}(-1))-3,847 \mathrm{e}-05 * \mathrm{D}(\operatorname{IMPMX}(-1))+$ $0,0107 * \mathrm{D}(\operatorname{MANUF}(-1))+0,1076$

$\mathrm{D}(\mathrm{IMPMX})=88,7759 *($ PRODINDEU $(-1)+0,0014 * \operatorname{IMPMX}(-1)-1,1598 *$ MANUF $(-1)$

$+1,5547)+126,8531 * \mathrm{D}($ PRODINDEU $(-1))-0,2243 * \mathrm{D}(\mathrm{IMPMX}(-1))-20,9112 *$

D $($ MANUF $(-1))+99,1241$ 
$\mathrm{D}($ MANUF $)=0,4056 *(\operatorname{PRODINDEU}(-1)+0,0014 * \operatorname{IMPMX}(-1)-1,1598 * \operatorname{MANUF}(-1)$

$+1,5547)+0,3398 * \mathrm{D}($ PRODINDEU $(-1))+0,000571 * \mathrm{D}(\operatorname{IMPMX}(-1))-0,4726 *$

D(MANUF(-1)) $+0,2395$

Nótese que los valores -0,0307 de la ecuación (2), 88,7759 de la ecuación (3) y 0,4056 de la ecuación (4), nos dan las velocidades de ajuste del modelo de corrección de errores EC. Todos estos son diferentes de cero, con lo cual obliga a un cambio en la variable endógena cuando se altera el comercio con los Estados Unidos. En valor absoluto, la cifra mayor es de 88,7759 e indica la relación que hace el mayor esfuerzo para lograr el ajuste en el tiempo, mientras que la cifra menor es -0,0307 y corresponde a la estructura que hace el menor esfuerzo.

Las demás componentes que están en primeras diferencias $D[x(t)]=x(t)-x(t-1)$ corresponden a los ajustes de corto plazo, acciones de ajuste forzando un cambio en las variables endógenas, D(PRODINDEU), D(IMPMX), D(TOTAL), de modo que cuando se alcanza el equilibrio, todas se hacen cero, quedando solamente la relación EC de largo plazo.

En definitiva utilizaremos el modelo de Hamilton (1989) para capturar la dinámica del sector industrial mexicano que responda a las preguntas establecidas en la introducción de este estudio. En los trabajos publicados se asume en general que el crecimiento porcentual de la manufactura es lineal (o loglineal), es decir, el nivel de crecimiento oscila alrededor de una media. Sin embargo, demostraremos que esto no es necesariamente así, de hecho podemos afirmar que en algunos subsectores de la manufactura el comportamiento es no lineal. Una sencilla prueba de especificación nos permite identificar la presencia de una variable latente, que en nuestro caso debiera ser el comercio con los Estados Unidos, que supondremos no es observable y produce cambios de régimen entre los dos estados. Es una dinámica no lineal que dicta los períodos de crecimiento y contracción, siendo esto modelado por una cadena de Markov de dos estados.

La estrategia que seguiremos es hacer el análisis a partir de los dos estados, crecer/ decrecer, siendo la variable latente quien activa cada estado para cada uno de sus 21 subsectores. Con esto se logra entender la dinámica de los diferentes subsectores estimando sus tiempos de ajuste.

\section{MODELO}

En esta sección presentamos el modelo del estudio (su desarrollo completo está en el anexo B). El Indice de la Manufactura es una variable evolutiva del sector real, la que se denota por $Y_{t}$. Su tasa de crecimiento solo toma dos alternativas: es positiva $\left(\% Y_{t}>0\right)$ o es no positiva $\left(\% Y_{t} \leq 0\right)$. Estos son dos regímenes y en cada uno de ellos hay una media, $\mu_{1}$ (media del estado de crecimiento) y $\mu_{2}$ (media del estado de decrecimiento).

Vamos a modelar la brecha entre el "crecimiento actual contra su crecimiento esperado", denotada por $\left[\% Y_{t}-\mu_{j}\right]$. Esta comparación indica la evolución del sector, cuando la brecha es grande se altera el ritmo productivo, capturado por medio de un 
modelo $A R(p)$. Así, cuando la variable latente se activa $S_{t}=1$ se presenta el régimen de crecimiento $\mathrm{m}\left(S_{t}=1\right)=\mu_{1}$, de otro modo se presenta el decrecimiento $S_{t}=2$, esto es: $\mu\left(S_{t}=2\right)=\mu_{2}$. En la literatura un modelo de cadenas de Markov con s-estados se denota por $M S w(s)$ (Markov Switching Model, orden s).

La prueba de especificación se basa en el hecho que si $\mu\left(S_{t}=1\right)=\mu_{1}=\mu_{2}=$ $\mu\left(S_{t}=2\right)$ significa que no se presenta la variable latente. Como observaremos, esto va a pasar en varias componentes de la manufactura. Es importante mencionar que cada nivel de $\mu\left(S_{t}=1\right), \mu\left(S_{t}=2\right)$ no solo está asociado a un nivel de actividad productiva, sino también a un volumen de empleo.

Para estudiar la dinámica manufacturera se aplica la siguiente especificación:

$$
\operatorname{MSw}(\mathrm{s})-\mathrm{AR}(\mathrm{p}) \% Y_{t}-\mu\left(S_{t}\right)=\phi_{1}\left(\% Y_{t-1}-\mu\left(S_{t}\right)\right)+\ldots+\phi_{p}\left(\% Y_{t-p}-\mu\left(S_{t}\right)\right)+\varepsilon_{t}
$$

Al transcurrir el tiempo, $S_{t}$ va tomando valores del conjunto $\{1,2\}$, marcando el régimen que impera en el sistema. El término error se supone ruido blanco y los parámetros $\varphi_{1}, \ldots, \varphi_{p}$ van a ser estimados, así como $\mu\left(S_{t}=1\right), \mu\left(S_{t}=2\right)$.

Los valores que indican la probabilidad de cada estado se escriben en términos de la matriz: $T=\left[\begin{array}{ll}p 11 & p 21 \\ p 12 & p 22\end{array}\right]$, llamada la matriz de transición de la cadena de Markov con dos estados. $P i j$ es la probabilidad de que estando en el estado $i$-ésimo se pase al estado $j$-ésimo. Sus columnas suman uno.

Una forma de interpretar el modelo es que el mecanismo de mercado tiene fallas, es decir, las señales no llegan de manera clara. Cada unidad productiva decide con información incompleta y racionalidad acotada, con lo cual esto repercute el nivel de producción. Se busca capturar la existencia de la falla por medio de la variable latente que dicta el estado $S_{t}$ del sistema, lo cual altera la brecha $\% Y_{t}-\mu\left(S_{t}\right)$. La falla se revela cuando la brecha es persistentemente grande y modifica el ritmo productivo. Los ajustes de la producción no se dan de manera suave sino súbita, repercutiendo las expectativas de ampliación de la planta, solicitándose más o menos créditos, nuevas o la dilatación de compras de maquinarias, compras o ventas de terrenos para nuevas plantas, asignación o reducción de presupuesto para I\&D, contratación de personal o despidos, nuevas inversiones en capacitación o reducciones de estas, etcétera.

El trabajo desarrollado por Hamilton (1989) permite responder a las preguntas mencionadas en la introducción del estudio:

A) Cuando la variable está creciendo, lo hace con un valor medio de:

$$
\mu\left[S_{t}=1\right]=\mu 1 \text {. }
$$

B) De modo similar, cuando decrece va a manifestarse con un valor medio de:

$$
\mu\left[S_{t}=2\right]=\mu 2 \text {. }
$$


C) La probabilidad de que la variable siga creciendo es P11.

D) $\mathrm{Al}$ estar creciendo una estimación de la duración de esta expansión está dada por $\mathrm{E}[\mathrm{P} 11]=1 /(1-\mathrm{P} 11)$ meses.

E) La probabilidad de que una variable que está creciendo pase al otro estado es P12.

E) De modo similar, la probabilidad de que si la variable decrece, entonces siga decreciendo es P22.

F) Al estar decreciendo, una estimación de la duración de la contracción es de $\mathrm{E}[\mathrm{P} 22]=1 /(1-\mathrm{P} 22)$ meses.

G) Las expansiones son más largas que las contracciones si E[P11]>E[P22].

H) La probabilidad de que si está decreciendo pase al otro estado es P21.

I) La probabilidad no condicional, de largo plazo, de que el sistema se encuentre en el estado $S_{t}=1$, o el $S_{t}=2$ es:

$$
\begin{aligned}
& \pi 1=P\left[S_{t}=1\right]=\frac{1-p 22}{2-p 11-p 22} \\
& \pi 2=P\left[S_{t}=2\right]=\frac{1-p 11}{2-p 11-p 22}
\end{aligned}
$$

J) La probabilidad no condicional de crecer es más grande que la de decrecer, si $\pi 1>\pi 2$.

\section{RESULTADOS}

En las estimaciones se usan datos de la INEGI para el período enero 1995 - junio 2012. Al respecto, se usan los datos sin ajustar por estacionalidad, con el propósito de tener la misma cifra que usan las empresas de cada sector para capturar la variable latente del modelo. En todos los casos el modelo se estima por máxima verosimilitud y obtiene convergencia, de modo que la función de verosimilitud está bien definida.

Se aplica la prueba de especificación $H_{0}: \mu 1=\mu 2$. Si no se rechaza $\mathrm{H}_{0}$, se toma la formulación lineal y su tratamiento es el de un $\mathrm{AR}(\mathrm{p})$ usual. No se presentan estos resultados, debido a que esta técnica ya es conocida, en cambio el interés se concentra en la no linealidad de algunos subsectores.

Las 6 series que no presentan una variable latente son: Prendas de Vestir, Cuero, Petróleo, Plástico, Productos Metálicos y Otras Manufacturas. Por otra parte 16 series 
presentan esta dualidad latente en su desempeño: Manufactura, Alimentos, Bebidas y Tabaco, Insumos Textiles, Productos Textiles, Madera, Papel, Impresión, Química, No Metálicos, Metálica Básica, Maquinaria, Electrónicos, Equipo Eléctrico, Transporte y Muebles.

La tasa de crecimiento medio del estado $S_{t}=1$, o sea, $\mu_{1}$ ya anualizada en algunos casos, es de dos dígitos. Por otra parte, en varios subsectores las caídas son pronunciadas, pero poco frecuentes. En ocasiones la caída revela una componente estacional de un mercado que se reduce, especialmente en textiles. Al respecto, debemos destacar que tomamos en cuenta el período de "la gran recesión" 2007/2008, en que el modelo indica el daño al que fueron sometidos los subsectores en esos años.

Nuestros resultados indican que la serie Manufactura tiene una variable latente, ya que las pruebas rechazan las siguientes hipótesis nulas: $H_{0}: \mu_{1}=0, \mu_{2}=0$ y $H_{0}$ : $\mu_{1}=\mu_{2}$. Así, la matriz de transición (transpuesta) es:

$$
T=\left[\begin{array}{ll}
p 11 & p 21 \\
p 12 & p 22
\end{array}\right]=\left[\begin{array}{ll}
0,964 & 0,777 \\
0,036 & 0,223
\end{array}\right]
$$

Para la Manufactura tenemos:

A) Cuando la variable está creciendo lo hace con un valor medio de: $\mu\left[S_{t}=1\right]=0,578 \%$, que anualizado es $7,165 \%$.

B) De modo similar, cuando decrece se manifiesta con un valor medio de: $\mu\left[S_{t}=2\right]=-6,611$, o sea, $-55,989 \%$.

C) La probabilidad de que la variable continúe creciendo es $p 11=0,964$.

D) Una expansión tiene una duración $1 /(1-p 11)=27,477$ meses.

E) Debido a que la variable crece, tiene una probabilidad de que inicie el decrecimiento de $p 12=0,036$.

F) La probabilidad de que continúe la contracción es de $p 22=0,223$.

G) La duración de una contracción es de 1/(1-p22) = 1,287 meses.

H) La probabilidad de que si está decreciendo pase al otro estado, o sea, que ahora crezca, es de: $p 21=0,777$.

I) La probabilidad no condicional de que el sistema se encuentre en el estado $S_{t}=1$, o el $S_{t}=2$ es: 


$$
\begin{aligned}
& \pi 1=P\left[S_{t}=1\right]=\frac{1-p 22}{2-p 11-p 22}=0,955 \\
& \pi 2=P\left[S_{t}=2\right]=\frac{1-p 11}{2-p 11-p 22}=0,045
\end{aligned}
$$

Para los subsectores se resumen los resultados en las Tablas 3 a 8 .

La manufactura cuenta con 8 subsectores con marcado crecimiento, que cumplen la condición $0.9<p 11<1$, lo que quiere decir que es altamente probable que si están creciendo sigan creciendo. Este grupo puede pasar a ser "la locomotora de la manufactura" (Tabla 4).

Con relación a la pregunta de si los períodos de expansión son más largos que las contracciones, tenemos que esto ocurre en 11 subsectores, pero en otros 5 la contracción dura más tiempo que la expansión, requiriendo atención Alimentos, Bebidas y Tabaco, Papel, Química y No Metálicos (Tabla 5).

En la Tabla 8 vemos que la probabilidad no condicional de crecer es mayor a la de contraerse en 11 subsectores.

En resumen, este mosaico de no linealidades refleja una fuerte heterogeneidad en el sector industrial. Encontramos por ejemplo manufactura artesanal, producción en cadena, en serie, por lotes, por encargo, etc. Además se mezclan diferentes zonas geográficas y diferentes ciudades. Por otro lado, muchas veces la producción es

TABLA 3

\begin{tabular}{|c|c|c|c|}
\hline Crecimento & Observado & Estimado & Estimado \\
\hline Variable & Media simple & $\mu 1$ crecer & $\mu 2$ decrecer \\
\hline Manufactura & 0,243 & 0,578 & $-6,611$ \\
\hline Alimentos & 0,138 & 2,953 & $-0,923$ \\
\hline Beb. y Tab. & 0,336 & 6,279 & $-2,514$ \\
\hline Ins. Textil & 0,077 & 2,257 & $-24,935$ \\
\hline Prod. Textil & $-0,063$ & 2,290 & $-30,598$ \\
\hline Madera & 0,136 & 7,716 & $-9,325$ \\
\hline Papel & 0,336 & 5,023 & $-3,946$ \\
\hline Impresión & 0,267 & 4,317 & $-11,827$ \\
\hline Química & 0,153 & 5,807 & $-3,836$ \\
\hline No Metálicos & 0,172 & 6,248 & $-0,666$ \\
\hline Metal. Básica & 0,239 & 0,466 & $-4,312$ \\
\hline Maquinaria & 0,229 & 1,289 & $-19,018$ \\
\hline Electrónicos & 0,191 & 1,409 & $-17,143$ \\
\hline Equipo Eléctrico & 0,281 & 2,532 & $-2,013$ \\
\hline Transporte & 0,536 & 1,054 & $-20,012$ \\
\hline Muebles & $-0,086$ & 1,396 & $-50,760$ \\
\hline
\end{tabular}

CRECIMIENTO BAJO LA EXPANSION Y LA CONTRACCION

Fuente: Cálculos del autor. 
llevada a veces por un pequeño porcentaje de trabajadores altamente calificados en empresas familiares, en medio de acentuadas restricciones financieras por falta de información. Así, los empresarios deben hacer importantes esfuerzos para concretar los planes de producción.

TABLA 4

PROBABILIDAD DE CONTINUAR EL CRECIMIENTO

\begin{tabular}{|lr|}
\hline Variable & P11 \\
\hline Manufactura & 0,964 \\
Ins. Textil & 0,912 \\
Prod. Textil & 0,958 \\
Metal. Básica & 0,992 \\
Maquinaria & 0,950 \\
Electrónicos & 0,952 \\
Transporte & 0,989 \\
Muebles & 0,971 \\
Beb. y Tab. & 0,700 \\
Impresión & 0,679 \\
Alimentos & 0,509 \\
Madera & 0,408 \\
Papel & 0,151 \\
No Metálicos & 0,126 \\
Química & 0,052 \\
Equipo Eléctrico & 0,015 \\
\hline
\end{tabular}

Fuente: Cálculos del autor.

TABLA 5

COMPARACION: ¿ES MAS PROBABLE UNA EXPANSION QUE UNA CONTRACCION?

\begin{tabular}{|ll|}
\hline Variable & EP11>EP22 \\
\hline Manufactura & Verdadero \\
Alimentos & Falso \\
Beb. y Tab. & Falso \\
Ins. Textil & Verdadero \\
Prod. Textil & Verdadero \\
Madera & Verdadero \\
Papel & Falso \\
Impresión & Verdadero \\
Química & Falso \\
No Metálicos & Falso \\
Metal. Básica & Verdadero \\
Maquinaria & Verdadero \\
Electrónicos & Verdadero \\
Equipo Eléctrico & Verdadero \\
Transporte & Verdadero \\
Muebles & Verdadero \\
\hline
\end{tabular}

Fuente: Cálculos del autor. 


\section{TABLA 6}

MESES QUE SE ESPERA DURE UNA EXPANSION

\begin{tabular}{|c|c|c|c|}
\hline \multirow{2}{*}{ Variable } & Meses expansión & Seguir creciendo & Iniciar caída \\
\hline & EP11 & P11 & P12 \\
\hline Manufactura & 27,477 & 0,964 & 0,036 \\
\hline Alimentos & 2,036 & 0,509 & 0,491 \\
\hline Beb. y Tab. & 3,336 & 0,700 & 0,300 \\
\hline Ins. Textil & 11,348 & 0,912 & 0,088 \\
\hline Prod. Textil & 23,883 & 0,958 & 0,042 \\
\hline Madera & 1,688 & 0,408 & 0,592 \\
\hline Papel & 1,177 & 0,151 & 0,849 \\
\hline Impresión & 3,115 & 0,679 & 0,321 \\
\hline Química & 1,055 & 0,052 & 0,948 \\
\hline No Metálicos & 1,144 & 0,126 & 0,874 \\
\hline Metal. Básica & 121,962 & 0,992 & 0,008 \\
\hline Maquinaria & 20,135 & 0,950 & 0,050 \\
\hline Electrónicos & 20,729 & 0,952 & 0,048 \\
\hline Equipo Eléctrico & 1,015 & 0,015 & 0,985 \\
\hline Transporte & 92,234 & 0,989 & 0,011 \\
\hline Muebles & 34,061 & 0,971 & 0,029 \\
\hline
\end{tabular}

Fuente: Cálculos del autor.

\section{TABLA 7}

MESES QUE SE ESPERA DURE UNA CONTRACCION

\begin{tabular}{|c|c|c|c|}
\hline \multirow{2}{*}{ Variable } & Meses contracción & Seguir a la baja & Iniciar crecimiento \\
\hline & EP22 & $\mathrm{P} 22$ & $\mathrm{P} 21$ \\
\hline Manufactura & 1,287 & 0,223 & 0,777 \\
\hline Alimentos & 5,343 & 0,813 & 0,187 \\
\hline Beb. y Tab. & 6,576 & 0,848 & 0,152 \\
\hline Ins. Textil & 1,000 & 0,000 & 1,000 \\
\hline Prod. Textil & 1,679 & 0,404 & 0,596 \\
\hline Madera & 1,391 & 0,281 & 0,719 \\
\hline Papel & 1,315 & 0,240 & 0,760 \\
\hline Impresión & 1,052 & 0,049 & 0,951 \\
\hline Química & 1,512 & 0,339 & 0,661 \\
\hline No Metálicos & 7,496 & 0,867 & 0,133 \\
\hline Metal. Básica & 5,122 & 0,805 & 0,195 \\
\hline Maquinaria & 1,000 & 0,000 & 1,000 \\
\hline Electrónicos & 1,539 & 0,350 & 0,650 \\
\hline Equipo Eléctrico & 1,000 & 0,000 & 1,000 \\
\hline Transporte & 1,890 & 0,471 & 0,529 \\
\hline Muebles & 1,000 & 0,000 & 1,000 \\
\hline
\end{tabular}

Fuente: Cálculos del autor. 
TABLA 8

PROBABILIDAD NO CONDICIONAL DE LARGO PLAZO: P1 EXPANSION, P2 CONTRACCION

\begin{tabular}{|llcc|}
\hline Variable & $\mathrm{p} 1>\mathrm{p} 2$ & $\mathrm{p} 1$ & $\mathrm{p} 2$ \\
\hline Manufactura & Verdadero & 0,955 & 0,045 \\
Alimentos & Falso & 0,276 & 0,724 \\
Beb. y Tab. & Falso & 0,337 & 0,663 \\
Ins. Textil & Verdadero & 0,919 & 0,081 \\
Prod. Textil & Verdadero & 0,934 & 0,066 \\
Madera & Verdadero & 0,548 & 0,452 \\
Papel & Falso & 0,472 & 0,528 \\
Impresión & Verdadero & 0,748 & 0,252 \\
Química & Falso & 0,411 & 0,589 \\
No Metálicos & Falso & 0,132 & 0,868 \\
Metal. Básica & Verdadero & 0,960 & 0,040 \\
Maquinaria & Verdadero & 0,953 & 0,047 \\
Electrónicos & Verdadero & 0,931 & 0,069 \\
Equipo Eléctrico & Verdadero & 0,504 & 0,496 \\
Transporte & Verdadero & 0,980 & 0,020 \\
Muebles & Verdadero & 0,971 & 0,029 \\
\hline
\end{tabular}

Fuente: Cálculos del autor.

\section{CONCLUSIONES}

Podemos interpretar los resultados del modelo de la siguiente forma. Usando la nomenclatura financiera, podemos decir que la manufactura y sus subsectores no constituyen un mercado profundo: decisiones de una o pocas empresas (locales o foráneas) alteran todo el volumen de producción. Esta dinámica de freno-arranque que origina la existencia de los dos niveles productivos, conlleva a pronunciadas oscilaciones en los niveles de actividad con un efecto perverso para el crecimiento del empleo en México.

La manufactura debe incorporar procesos de innovación y con mayor valor agregado, que permitan lograr un estado de crecimiento medio mayor y por otra parte ampliar la capacidad negociadora para tener períodos más cortos de contracción. Así, productos con mayor valor agregado permitirán períodos más largos de crecimiento.

Al respecto, para lograr tasas de crecimiento mayores se pueden seguir las siguientes recomendaciones:

a) Los subsectores requieren que las respectivas asociaciones de productores (las llamadas Cámaras) tengan un papel activo en el desarrollo del sector utilizando la ley de la ciencia y tecnología.

b) Los productos deben tener una reputación de marca en los mercados.

c) La publicidad debe ser compartida, lo que disminuye los costos. Por ejemplo, el gobierno puede aprovechar a las delegaciones comerciales de las embajadas para publicidad.

d) Reducir el impacto de los monopolios, ya que una sola empresa produce más inestabilidad. 
e) Mejorar la eficiencia en las políticas de gobierno.

f) Mejorar los convenios internacionales, para asegurar la inversión extranjera directa en el sector industrial.

g) Mejorar las ventajas comparativas y el comercio internacional.

h) Aprovechar las transferencias tecnológicas con el exterior, ya que:

- La ciencia y tecnología conduce a mayores ventas.

- Las ventas, internas y de exportación, conducen a mayores ganancias.

- Las ganancias conducen a buscar en la tecnología más aplicaciones de ideas con marcado valor agregado en los productos.

\section{BIBLIOGRAFIA}

ACEVEDO, F.E. (2002). "Causas de la recesión en la industria maquiladora”, Momento Económico 124, pp. 11-25.

COSSLETT, S. y L-F. LEE (1985). "Serial Correlation in Latente Discrete Variable Models", Journal of Econometrics 27, pp. 79-97.

CUADRA, G. (2008). "Hechos Estilizados del Ciclo Económico en México", Documento de Investigación 2008-14, Banco de México.

DEMPSTER, A.P., N.M. LAIRD y D.B. RUBIN (1977). "Maximum Likelihood from Incomplete Data via the EM Algorithm", Journal of the Royal Statistical Society, Series B 39, pp. 1-38.

DUSSEL PETERS, E. (2009). "La Manufactura Mexicana ¿opciones de recuperación?", Economía Informa 357.

FEDERAL RESERVE BANK. http://research.stlouisfed.org/fred2/ en la fecha 24/08/2012.

FUJI, G. (2000). "El comercio exterior manufacturero y los límites al crecimiento económico de México", Comercio Exterior, noviembre, pp. 1008-1014.

HAMILTON, J. (1989). "A New Approach to the Economic Analysis of Nonstationary Times Series and the Business Cycles", Econometrica 57, pp. 357-384.

HAMILTON, J. (1994). Time Series Analysis, Princeton University Press.

INEGI. http://www.inegi.org.mx/sistemas/bie/ en la fecha 24/08/2012.

MEJIA-REYES, P. y J. CAMPOS-CHAVEZ (2011). "Are the Mexican States and the United States Business Cycles Synchronized? Evidence from the Manufacturing Production", Economía Mexicana Nueva Epoca XX (1), primer semestre de 2011, pp. 79-112.

MENDOZA COTA, E. (2008). "Integración económica y sincronización del ciclo económico entre México y Estados Unidos", Boletín de indicadores y pronósticos económicos de la frontera norte 31, Christian de la Luz Tovar, responsable, El Colegio de la Frontera Norte, Tijuana, julio de 2008.

MORENO VILLANUEVA, L. y A. PALERM VIQUEIRA (2001). "Comercio intraindustrial en el sector manufacturero mexicano", Comercio Exterior, septiembre, pp. 789-794.

RESERVA FEDERAL. http://research.stlouisfed.org/fred2/ en la fecha 24/08/2012.

UNGER, K. (1993). "Productividad, desarrollo tecnológico y competitividad exportadora en la industria mexicana", Economía Mexicana Nueva Epoca II (1), enero-julio 1993, pp. 183-237. 


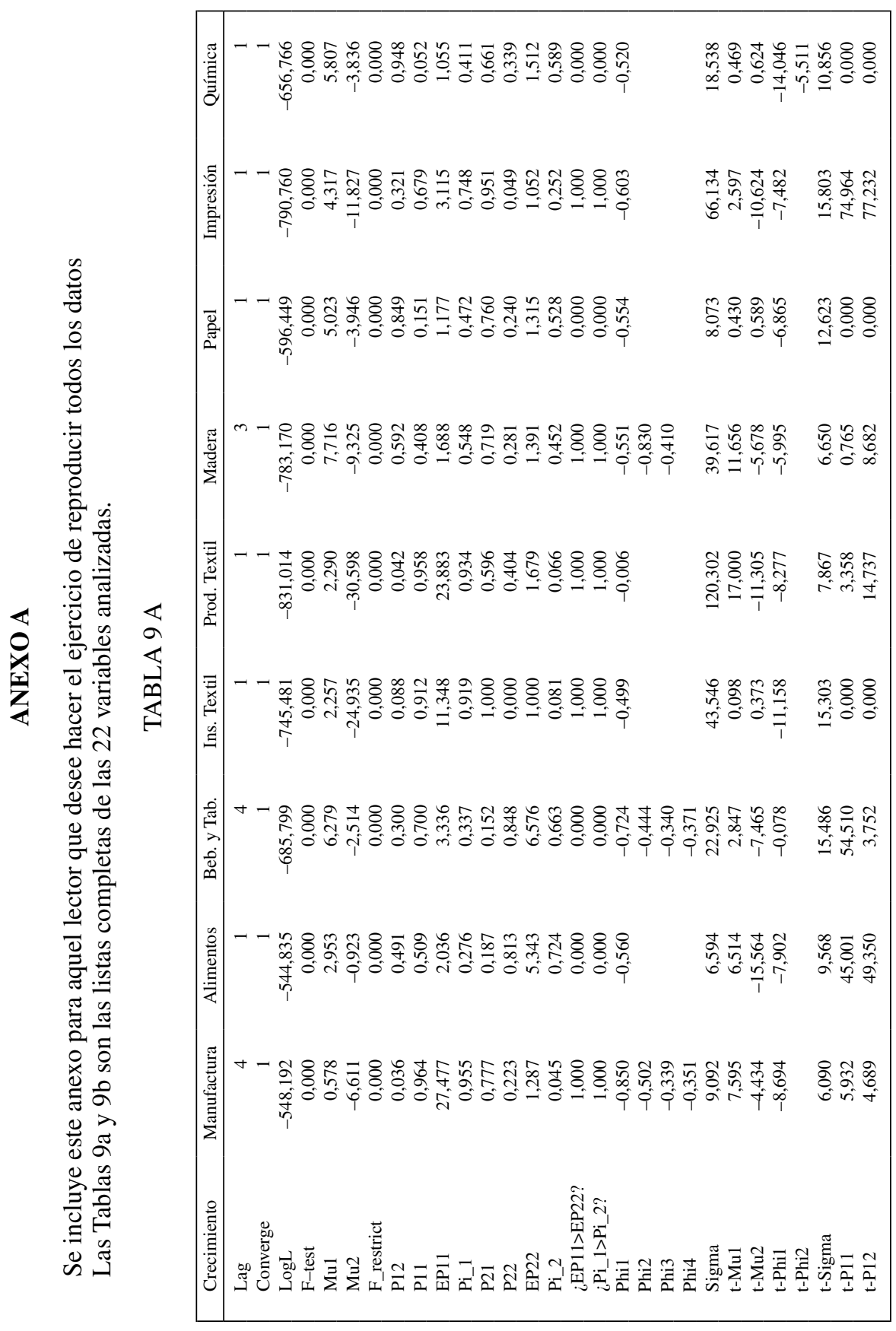




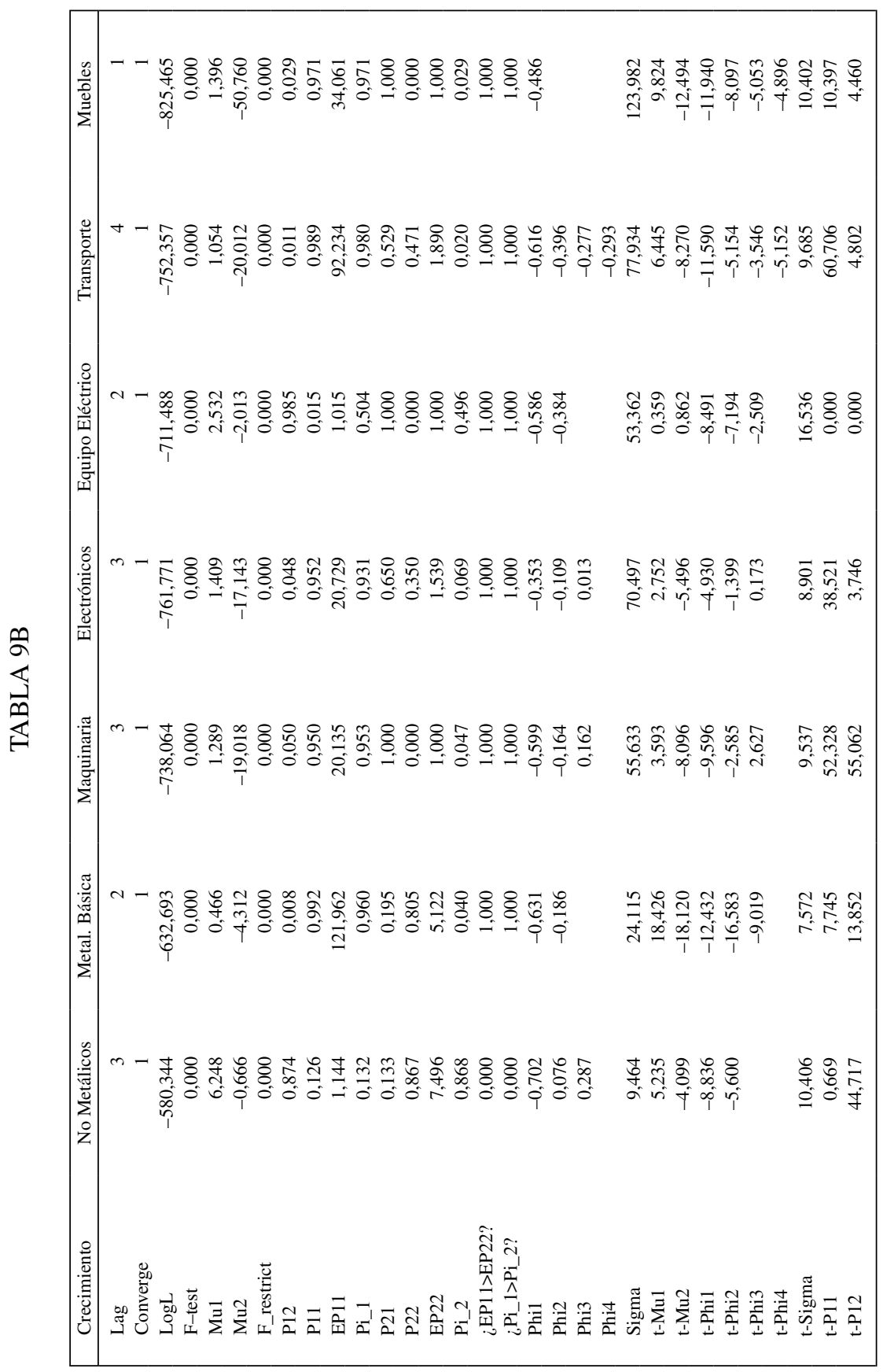




\section{ANEXO B}

El modelo se basa en varios desarrollos: la teoría de las cadenas de Markov, mezcla de distribuciones, econometría bayesiana y el método EM. Una explicación detallada está en el libro de Hamilton (1989 y 1994, Capítulo 22).

Cadenas de Markov. Tenemos un proceso estocástico que tiene dos estados y el objetivo es explicar la transición entre ambos estados. Se le llama una Cadena de Markov cuando la transición, es decir, el cambio de un estado a otro, solo depende del estado actual, cumpliendo con la condición $p i j=P\left[S_{t}=j \mid S_{t-1}=i\right](i-->j)$.

La probabilidad de estar en el estado $S_{t}=j$ solo depende del estado $S_{\mathrm{t}-1}=\mathrm{i}$, por lo que es independiente de los valores de los estados $S_{t-2}, S_{t-3}, \ldots, S_{0}$. Las probabilidades no dependen del tiempo, por lo que se llama una cadena homogénea.

Como pij son probabilidades, deben satisfacer $p i j \geq 0$ y también $p i 1+p i 2=1$ para $i=1,2$; las entradas de la matriz son no negativas y cada columna suma uno.

La matriz de transición es $\quad T=\left[\begin{array}{ll}p 11 & p 21 \\ p 12 & p 22\end{array}\right]=\left[\begin{array}{cc}p 11 & 1-p 22 \\ 1-p 11 & p 22\end{array}\right]$

Si la distribución de probabilidad de los estados es $p(0)=(p 1(0), p 2(0))^{t}$ significa que $p 1(0)$ es la probabilidad de que en el momento 0 se active el estado 1 y tiene una probabilidad de $p 1(0)$; mientras que $p 2(0)$ es la probabilidad de que sea el estado 2 el que esté activo al momento inicial. Cumplen con la condición $p 1(0)+p 2(0)=1$, que significa que al momento cero está activo algún estado, ya sea el $S_{t}=1$ o $S_{t}=2$.

La distribución de probabilidad de los estados se modifica por medio de una multiplicación de la matriz de transición por el vector de estados:

$$
p(1)=T \cdot p(0)=\left(\begin{array}{l}
p 1(1) \\
p 2(1)
\end{array}\right)=\left[\begin{array}{ll}
p 11 & p 21 \\
p 12 & p 22
\end{array}\right]\left(\begin{array}{l}
p 1(0) \\
p 2(0)
\end{array}\right)=\left(\begin{array}{l}
p 1(0) p 11+p 2(0) p 21 \\
p 1(0) p 12+p 2(0) p 22
\end{array}\right)
$$

siendo p(1) la probabilidad de los estados del siguiente período. De hecho se tiene la interesante relación para las cadenas de Markov homogéneas:

$$
\left(\begin{array}{l}
p 1(n+1) \\
p 2(n+1)
\end{array}\right)=\left[\begin{array}{ll}
p 11 & p 21 \\
p 12 & p 22
\end{array}\right]\left(\begin{array}{l}
p 1(n) \\
p 2(n)
\end{array}\right)=T^{n+1}\left(\begin{array}{l}
p 1(0) \\
p 2(0)
\end{array}\right)
$$

En nuestro caso interesa la evolución del estado $1 \xi_{t}=\left[\begin{array}{l}1 \\ 0\end{array}\right]$ o del estado 2 $\xi_{t}=\left[\begin{array}{l}0 \\ 1\end{array}\right]$, que tiene las expectativas condicionales: 


$$
\begin{aligned}
& E\left[\xi_{t} \mid S_{t-1}=1\right]=\left[\begin{array}{l}
p 11 \\
p 12
\end{array}\right] \\
& E\left[\xi_{t} \mid S_{t-1}=2\right]=\left[\begin{array}{l}
p 21 \\
p 22
\end{array}\right]
\end{aligned}
$$

De modo que la expectativa condicional que marca el cambio entre los estados está dada por la matriz de transición T, de modo que $E\left[\xi_{t+1} \mid \xi_{t}\right]=T \xi_{t}$.

La variable latente que corresponde a los estados sigue un proceso $\mathrm{AR}(1)$ :

$$
\xi_{t+1}=T \xi_{t}+v_{t+1}
$$

Explícitamente tenemos:

$$
\left[\begin{array}{c}
\xi 1, t+1 \\
1-\xi 1, t+1
\end{array}\right]=\left[\begin{array}{cc}
p 11 & 1-p 22 \\
1-p 11 & p 22
\end{array}\right]\left[\begin{array}{c}
\xi 1, t \\
1-\xi 1, t
\end{array}\right]+\left[\begin{array}{c}
v_{1, t+1} \\
v_{2, t+1}
\end{array}\right]
$$

obteniendo del primer renglón:

$$
\xi_{1, t+1}=(1-p 22)+(-1+p 11+p 22) \xi_{1, t}+v_{t+1}
$$

Para que este proceso $\mathrm{AR}(1)$ sea estacionario, se requiere que: $-1<-1+p 11+$ $p 22$, lo cual es equivalente a la condición:

$$
0<p 11+p 22<2
$$

Se cumple si se sabe que $p 11=1$ o $p 22=1$, pero no ambos. Si $p i i=1$ se le llama al estado i un estado absorbente, ya que si la variable latente toma el valor $S_{t}=i$ va a permanecer allí con probabilidad igual a uno. En nuestro caso, $p 11$ y p22 tienen aplicaciones importantes.

Además su media no condicional es:

$$
E\left[\xi_{1, t}\right]=\frac{1-p 22}{2-p 11-p 22}
$$


Por otra parte, como: $\xi_{2, t}=1-\xi_{1, t}$, tenemos que:

$$
E\left[\xi_{2, t}\right]=1-\frac{1-p 22}{2-p 11-p 22}=\frac{1-p 11}{2-p 11-p 22}
$$

Lo que nos interesa es la posición de equilibrio de largo plazo, donde la distribución de probabilidades de los estados ya no se modifica y corresponde a un vector propio con valor propio unitario, o sea, el vector de equilibrio satisface $T \pi=\pi$, habiendo una distribución de probabilidad de los estados que es invariante y se le llama el vector de probabilidades ergódicas. Se puede obtener mediante el concepto de límite, ya que la invariancia implica que los diferentes estados tengan la misma distribución de probabilidad.

$$
\lim _{n \rightarrow \infty} T^{n}=\left[\begin{array}{ll}
\pi 1 & \pi 1 \\
\pi 2 & \pi 2
\end{array}\right]
$$

Tiene la interesante cualidad de ser la expectativa no condicional de la variable latente.

$$
\pi=E\left[\xi_{t}\right]
$$

Así se puede calcular este vector de equilibrio:

$$
\pi=\left[\begin{array}{c}
\Pi_{1} \\
\Pi_{1}
\end{array}\right]=\left[\begin{array}{c}
\frac{1-p 22}{2-p 11-p 22} \\
\frac{1-p 11}{2-p 11-p 22}
\end{array}\right]
$$

En suma, la probabilidad no condicional de que en el largo plazo el sistema se encuentre en el estado 1 o el 2 es:

$$
\begin{aligned}
& P\left[S_{t}=1\right]=\frac{1-p 22}{2-p 11-p 22} \\
& P\left[S_{t}=2\right]=\frac{1-p 11}{2-p 11-p 22}
\end{aligned}
$$


Si consideramos la variable Bernoulli (éxito- $p$, fracaso- $q$ ), la distribución geométrica se refiere al número de ensayos de Bernoulli requeridos hasta que se obtiene el primer éxito y tiene una media $1 /(1-q)$, donde $p=\operatorname{Prob}($ éxito), $p=(1-q)$, está dada por:

$$
\sum_{k=1}^{\infty} k q^{k-1}(1-q)=(1-q)^{-1}
$$

Tiene dos aplicaciones importantes:

A) Condicional a estar en una contracción, la duración esperada del período recesivo es $(1-p 22)^{-1}$, ya que se toma $q=p 22$ y $1-q=\mathrm{p} 21$.

B) Condicional a estar en una expansión, la duración esperada del crecimiento es $(1-p 11)^{-1}$, ya que se toma $q=p 11$ y $1-q=p 12$.

Necesitamos un método que realice la estimación para las entradas de la matriz de transición y para el vector de equilibrio $\pi$.

El método de estimación es el algoritmo EM, propuesto por Dempster, Laird y Rubin (1977), y es un método general para estimar sumas de distribuciones, llamadas mixture distributions. Se considera que el proceso observado es una suma de varias densidades y se estiman los parámetros de esta suma mostrando ser útil para variables latentes; se aplica en las cadenas de Markov y requiere del uso de estadística bayesiana, ya que juega un papel esencial para estimar la probabilidad posterior: debido a que se observa $Y_{t}$ deseo medir la probabilidad de que haya sido generada por el estado $S_{t}=j, j=1,2$. EM es un algoritmo iterativo que consiste de la etapa E (Expectativa) y del paso $\mathrm{M}$ (Maximizar).

Mezcla de distribuciones: Tenemos dos distribuciones cuando la variable $\% Y_{t}$ está bajo el régimen 1 , tiene una distribución $\% Y_{t} \sim N\left(\psi_{1}, \sigma_{1}^{2}\right)$ y cuando está bajo el régimen 2, sigue la distribución $\% Y_{t} \sim N\left(\psi_{2}, \sigma_{2}^{2}\right)$, los estados entre sí son independientes.

Note que:

$$
\begin{aligned}
E\left[\% Y_{t} \mid S_{t}=j, \% Y_{t-1}, \ldots, \% Y_{t-p}\right]=\psi_{j} \\
\quad=\mu\left(S_{t}\right)+\phi_{1}\left(\% Y_{t-1}-\mu\left(S_{t}\right)\right)+\ldots+\phi_{p}\left(\% Y_{t-p}-\mu\left(S_{t}\right)\right)
\end{aligned}
$$

El vector de parámetros a estimar es: $\theta=\left(\mu_{1}, \mu_{2}, \phi_{1}, \ldots, \phi_{p}, \sigma_{1}^{2}, \sigma_{2}^{2}, \pi_{1}, \pi_{2}\right)$.

Como deseamos poner en claro el método de estimación EM, vamos a seguir de cerca a Hamilton (1994, cap. 22). Deseamos estimar la constante, o sea, $E\left[\% Y_{t} \mid \% Y_{t-1}, \ldots \% Y_{t-p}\right]=\psi_{j}=\mu\left(S_{t}\right)$ y las probabilidades de largo plazo, por lo que 
la matriz de transición es omitida, así como el proceso que sigue la variable latente (Cosslett y Lee, 1985).

Tenemos las densidades condicionales, para los estados $j=1,2$ :

$$
f\left(\% Y_{t} \mid S_{t}=j ; \theta\right)=\frac{1}{\sqrt{2 \pi \sigma j}} \exp \left\{\frac{-\left(\% Y_{t}-\psi_{j}\right)^{2}}{2 \sigma_{j}^{2}}\right\}
$$

El vector de parámetros se ha reducido: $\theta=\left(\mu_{1}, \mu_{2}, \sigma_{1}^{2}, \sigma_{2}^{2}, \pi_{1}, \pi_{2}\right)$.

El régimen $S_{t}$ tiene probabilidad no condicional: $P\left(S_{t}=j ; \theta\right)=\pi_{j}$.

$\% Y$ tiene como distribución conjunta:

$P\left(\% Y_{t}, S_{t}=j ; \theta\right)=f\left(\% Y_{t} \mid S_{t}=j ; \theta\right) P\left[S_{t}=j ; \theta\right]=\frac{\pi_{j}}{\sqrt{2 \pi \sigma_{j}}} \exp \left\{\frac{-\left(\% Y_{t}-\mu_{j}\right)^{2}}{2 \sigma_{j}^{2}}\right\}$

La densidad no condicional se obtiene sumando sobre los dos regímenes considerados.

$$
\begin{aligned}
& f\left(\% Y_{t}, \theta\right)=P\left(\% Y_{t}, S_{t}=1 ; \theta\right)+P\left(\% Y_{t}, S_{t}=2 ; \theta\right)= \\
& =\frac{\pi_{1}}{\sqrt{2 \pi \sigma_{1}}} \exp \left\{\frac{-\left(\% Y_{t}-\mu_{1}\right)^{2}}{2 \sigma_{1}^{2}}\right\}+\frac{\pi_{2}}{\sqrt{2 \pi \sigma_{2}}} \exp \left\{\frac{-\left(\% Y_{t}-\mu_{2}\right)^{2}}{2 \sigma_{2}^{2}}\right\}
\end{aligned}
$$

Para estimar por máxima verosimilitud la función que debemos considerar es:

$$
\begin{aligned}
& \mathscr{L}(\theta)=\sum_{t=1}^{T} \log f\left(\% Y_{t}, \theta\right) \\
& \quad=\sum_{t=1}^{T} \log \left\{\frac{\pi_{1}}{\sqrt{2 \pi \sigma_{1}}} \exp \left\{\frac{-\left(\% Y_{t}-\mu_{1}\right)^{2}}{2 \sigma_{1}^{2}}\right\}+\frac{\pi_{2}}{\sqrt{2 \pi \sigma_{2}}} \exp \left\{\frac{-\left(\% Y_{t}-\mu_{2}\right)^{2}}{2 \sigma_{2}^{2}}\right\}\right\}
\end{aligned}
$$

sujeta a las restricciones $\pi_{1}+\pi_{2}=1 \pi_{1}, \pi_{2} \geq 0$. 
El uso de la estadística bayesiana es en el paso E para estimar la probabilidad de estar en el régimen 1 o 2 . Es la probabilidad posterior de que la observación $\% Y_{t}$ pertenece al régimen $S_{t}=j$, debido al vector de parámetros $\theta$

$$
P\left[S_{t}=j \mid \% Y_{t} ; \theta\right]=\frac{P\left[\% Y_{t}, S_{t}=j ; \theta\right]}{f\left(\% Y_{t} ; \theta\right)}=\frac{\pi_{j} f\left(\% Y_{t} \mid S_{t}=j ; \theta\right)}{f\left(\% Y_{t} ; \theta\right)}
$$

Entonces se estima la probabilidad, debido a la observación $\% Y_{t}$, de que el régimen $j$ es el responsable de generar esta observación.

$\pi_{j}=P\left(S_{t}=j ; \theta\right)$ es la probabilidad prior, $f\left(\% Y_{t} \mid S_{t}=j ; \theta\right)$ es la densidad condicional, la probabilidad posterior $P\left[S_{t}=j \mid \% Y_{t} ; \theta\right]$ es y se busca que sea lo más elevada posible.

Luego se utiliza la relación de Bayes:

$$
\begin{gathered}
P\left(B_{j} \mid A\right)=\frac{P\left(B_{j}\right) P\left(A \mid B_{j}\right)}{\left(\sum_{j=1}^{s} P\left(B_{j}\right) P\left(A \mid B_{j}\right)\right)} \\
P(A)=\sum_{j=1}^{s} P\left(B_{j}\right) P\left(A \mid B_{j}\right)
\end{gathered}
$$

$\{B 1, B 2, \ldots, B s\}$ es una partición del espacio de eventos, en el modelo los regímenes $\left\{S_{1}, S_{2}\right\}$ también lo son.

En el paso M maximizar es donde se realiza y actualiza la estimación de los parámetros del método iterativo. Se obtienen los estimadores de máxima verosimilitud para $j=1,2$.

Fórmula A $\quad \hat{\mu}_{j}=\frac{\sum_{t=1}^{T} \% Y_{t} \cdot P\left[S_{t}=j \mid \% Y_{t} ; \hat{\theta}\right]}{\sum_{t=1}^{T} P\left[S_{t}=j \mid \% Y_{t} ; \hat{\theta}\right]}$

Fórmula B $\widehat{\sigma_{j}^{2}}=\frac{\sum_{t=1}^{T}\left(Y_{t-}-\hat{\mu}_{j}\right)^{2} \cdot P\left[S_{t}=j \mid \% Y_{t} ; \hat{\theta}\right]}{\sum_{t=1}^{T} P\left[S_{t}=j \mid \% Y_{t} ; \hat{\theta}\right]}$ 
Fórmula C $\quad \hat{\pi}_{j}=\frac{1}{T} \sum_{t=1}^{T} P\left[S_{t}=j \mid \% Y_{t} ; \hat{\theta}\right]$

El algoritmo EM se describe a continuación:

1. Se inicia con valores iniciales de todos los parámetros denotado por $\widehat{\theta(1)}$.

2. Se aplican conceptos bayesianos y se obtiene una estimación para $P\left[S_{t}=j \mid \% Y_{t} ; \hat{\theta}(1)\right]$.

3. La probabilidad obtenida se utiliza en las fórmulas A, B, C, para realizar una estimación de $\theta=\left(\mu_{1}, \mu_{2}, \sigma_{1}^{2}, \sigma_{2}^{2}, \pi_{1}, \pi_{2}\right)$.

4. Obteniendo un nuevo estimador $\widehat{\theta(2)}$, se puede regresar al paso 2 .

5. Nuevamente se aplica la fase $\mathrm{M}$ al reestimar $\theta$ y obtener $\widehat{\theta(3)}$.

6. Se continúa pasando de $\widehat{\theta(n)}$ a $\widehat{\theta(n+1)}$ y se detiene el ciclo cuando la distancia es pequeña, digamos $[\widehat{\theta(n)}, \theta \widehat{(n+1)}]<0,00001$.

Un defecto que tiene el método EM es que no hay nada que garantice que el máximo sea único, tampoco que el algoritmo converja, ya que la función de máxima verosimilitud pudiera tener varios máximos o ser plana en regiones.

Una vez que los parámetros se han estimado, se construye el suavizamiento de Kim, que permite visualizar con facilidad la probabilidad de que al momento $t$ la variable $\% Y_{t}$ esté decreciendo. Esto es necesario, ya que la serie que describe la probabilidad de que la variable se contraiga tiene un zigzag muy pronunciado, por lo que se requiere el suavizamiento para tomar las fluctuaciones importantes, pero eliminando el exceso de zigzag. 